\title{
Seasonal variation in community-acquired pneumonia
}

\author{
D. Lieberman*+, D. Lieberman+, A. Porath ${ }^{+}$
}

Seasonal variation in community-acquired pneumonia. D. Lieberman, D. Lieberman, A. Porath. (O) ERS Journals Ltd 1996.

ABSTRACT: Seasonal variation in the incidence of disease in general, and of infectious diseases in particular, is vitally important information for epidemiologists and for clinicians who deal with these diseases. In relation to communityacquired pneumonia (CAP), the seasonal distribution of the disease and, in particular, of each of the specific aetiological agents is important.

All 346 adult patients who were admitted to a regional hospital in southern Israel for CAP over a period of 1 year were included in a prospective study. As part of the study, a comprehensive diagnostic work-up was performed to identify the specific causative agents for CAP. The study findings were analysed and are presented in terms of the seasonal distribution of the disease.

Thirty four percent of CAP cases occurred in the spring and only $18 \%$ in the autumn $(p=0.036)$. Respiratory viruses CAP were predominant in the winter and spring ( $\mathrm{p}=0.009)$, whilst Mycoplasma pneumoniae CAP reached its peak incidence of $41 \%$ in the spring compared with $15 \%$ during the winter months $(p=0.054)$. No significant seasonal predominance was observed for the other specific aetiological agents of CAP. In 58\% of the CAP patients who were hospitalized in the autumn we identified a pneumococcal aetiology, whilst this agent was found in only $32 \%$ of the cases in the winter.

In contrast with popular public opinion, winter with its low temperatures is not the main reason for the development of community-acquired pneumonia. Most of the specific aetiologies for community-acquired pneumonia, with the exception of respiratory viruses and Mycoplasma pneumoniae, have no seasonal predilection.

Eur Respir J., 1996, 9, 2630-2634.
*The Pulmonology Unit and the Division of Internal Medicine, the Soroka Medical Center of Kupat Holim Klalit and the Faculty of Health Sciences, Ben-Gurion University of the Negev, Beer-Sheva, Israel.

Correspondence: D. Lieberman

Pulmonary Unit

Soroka Medical Center

Beer-Sheva

Israel 84101

Keywords: Community-acquired pneumonia, Israel

seasonal variation

Received: April 291996

Accepted after revision August 251996
Community-acquired pneumonia (CAP) is a common infectious disease. Like other infectious diseases, its seasonal variation has epidemiological significance. Despite the high prevalence of CAP and the importance of its seasonal distribution, this subject has not been reported often in the literature. Only a minority of studies on CAP have related to the seasonal incidence of the disease [1-3].

In a comprehensive prospective study of 346 adult patients, who were hospitalized with CAP in the Soroka Medical Center in Beer-Sheva, Israel over the course of 1 year, we had the opportunity to determine the seasonal incidence of CAP and of each of its specific aetiological agents. The purpose of this paper is to describe and discuss this seasonal variation.

The Soroka Medical Center in Beer-Sheva is located in a city of 150,000 residents in the south of Israel. It serves a population of 300,000 inhabitants of the Negev region, a semi-arid desert area, mainly at sea level. Average temperatures during the study year ranged from $27^{\circ} \mathrm{C}$ in the summer to $9^{\circ} \mathrm{C}$ in the winter. The average yearly rainfall (which falls exclusively during the winter) is $200 \mathrm{~mm}$.
Materials and methods

\section{Patients}

We conducted a prospective study of the cause of CAP in 346 adult patients who were admitted to the hospital with this diagnosis at the Soroka Medical Center in Beer-Sheva, Israel over the period of 1 year between November 1, 1991 and October 31, 1992. The study was approved by the review board for human research (the Helsinki committee) of the Soroka Medical Center, and all patients gave their informed consent to participate.

The mean (SD) age of the patients was 49 (20) yrs (range 17-94 yrs). One hundred and eighty seven patients $(54 \%)$ were male. Sixteen patients $(5 \%)$ died in the hospital. All other patients were alive at least 6 weeks after admission to the hospital. During the course of their hospitalization, the patients were diagnosed and treated by the medical staff of the internal medicine wards, without intervention by the investigators. At discharge, the patients were referred to the investigators 
at the pulmonary disease clinic of the hospital, for clinical and radiological follow-up.

CAP was diagnosed by the presence of an acute febrile disease, with an acute pulmonary infiltrate on chest radiogram, and a clinical and radiological course that confirmed the diagnosis. Exclusion criteria included patients with positive blood tests for human immunodeficiency virus (HIV), patients with lung malignancies, and patients who were discharged from the hospital less than 21 days before their present admission to the hospital with pneumonia.

\section{Causal diagnoses}

In addition to routine hospital blood tests (complete blood count, biochemistry and blood cultures), a serum sample was obtained within the first $48 \mathrm{~h}$ of admission for serological testing. A second (convalescence) serum was obtained from 308 patients (89\%), usually at the follow-up appointment. The mean (SD) interval between the two serum samples was 32 (12) days (range 17-45 days). All sera were separated immediately and stored at $-70^{\circ} \mathrm{C}$ until tested.

The diagnosis of bacterial pathogens was based on positive blood cultures and/or positive serological tests. In patients with a positive blood or pleural fluid culture, the isolated bacterium was considered to be the causative agent of CAP. In order to identify possible bacterial pathogens in patients with negative blood cultures, 308 serum pairs were tested for the presence of specific antibodies to Streptococcus pneumoniae pneumolysin, Haemophilus influenzae and Moraxella (Branhamella) catarrhalis. Immunoglobulin $\mathrm{G}$ ( $\mathrm{IgG}$ ) antibodies to pneumococcal protein toxin, pneumolysin, were measured by enzyme immunoassay (EIA) utilizing pneumolysin produced in Bacillus subtilis as antigen [4]. A rise in antibody titre equal to or more than twofold between paired sera was considered diagnostic for S. pneumoniae infection $[4,5]$. Total antibodies to unencapsulated $H$. influenzae [6] and $M$. catarrhalis [7, 8] were measured by EIA, using whole bacterial cells of respective bacteria as antigens. A rise in antibody titre of $\geq 3$ between paired sera was considered to be diagnostic.

$S$. pneumoniae specific immune complexes were determined in all 654 (paired and unpaired) sera, by measuring antibodies to pneumolysin and to the mixture of 23 capsular polysaccharides present in vaccine, from precipitated and redissolved immune complexes $[9,10]$. The cut-off value for the presence of $S$. pneumoniae immune complexes was based on results of serum samples from 40 healthy elderly people (mean $\pm 2 \mathrm{SD}$ ). All bacterial serological tests were conducted at the Finnish National Public Health Institute in Helsinki and in Oulu, Finland.

CAP was considered to be caused by $S$. pneumoniae if there was a positive culture for S. pneumoniae (blood or pleural fluid), or a positive serological diagnosis for $S$. pneumoniae based on pneumolysin antibodies or the presence of $S$. pneumoniae specific immune complexes, as detailed above. CAP was determined to be caused by $H$. influenzae or $M$. catarrhalis in the presence of a positive blood culture or positive serology.

Serological testing was used to identify Mycoplasma pneumoniae, Chlamydia pneumoniae, Legionella spp.,
Coxiella burnetti and six respiratory viruses (influenza $\mathrm{A}$, influenza $\mathrm{B}$, adenovirus, respiratory syncytial virus (RSV), parainfluenza 1 and parainfluenza 3). Details of the various serological methods used and of the criteria for positive serological diagnoses for each of these causative agents have been published previously [11].

\section{Meteorological data}

Measurements of mean daily temperatures and relative humidities for the study months were conducted at the Beer-Sheva meteorological station of the meteorological service of the Israel Ministry of Transportation.

\section{Data analysis}

The date of hospitalization was used as the time of occurrence of pneumonia in this study. To analyse the seasonal incidence of CAP, the months of September, October and November were defined as autumn, December, January and February as winter, March, April and May as spring, and June, July and August as summer. The Chi-squared test was used to determine the significance of differences in incidence between the seasons. The null hypothesis of the study was that there were no seasonal differences in the incidence of CAP. A p-value of $<0.05$ was considered significant.

\section{Results}

Table 1 shows the frequency distribution of the various causal agents for CAP among the 346 patients hospitalized with this diagnosis during the course of the study year. In all, the causal agent was identified in 279 patients $(81 \%)$. A single aetiology was found in 146 patients $(42 \%)$, while more than one aetiological agent was found in 133 patients (38\%).

The aetiological frequencies by age and a discussion of the incidence of multiple aetiologies for CAP can be found in our previous publication on these subjects [11].

Table 1. - Frequency distribution of community-acquired pneumonia (CAP) aetiologies in 346 patients

\begin{tabular}{lrr}
\hline & \multicolumn{2}{c}{ Patients } \\
\cline { 2 - 3 } Pathogen & $\mathrm{n}$ & $\%$ \\
\hline Pneumococcus & 148 & 43 \\
Mycoplasma pneumoniae & 101 & 29 \\
Chlamydia pneumoniae & 62 & 18 \\
Legionella spp. & 56 & 16 \\
Viruses & 35 & 10 \\
Coxiella burnetii & 20 & 6 \\
Haemophilus influenzae & 19 & 6 \\
Other* & 21 & 6 \\
Unknown aetiology & 67 & 19 \\
\hline
\end{tabular}

*Including Moraxella catarrhalis (7), active pulmonary tuberculosis (7), nonpneumoniae streptococcus (4), Staphylococcus aureus (1), Acinetobacter spp. (1), and Pseudomonas aeruginosa (1). 


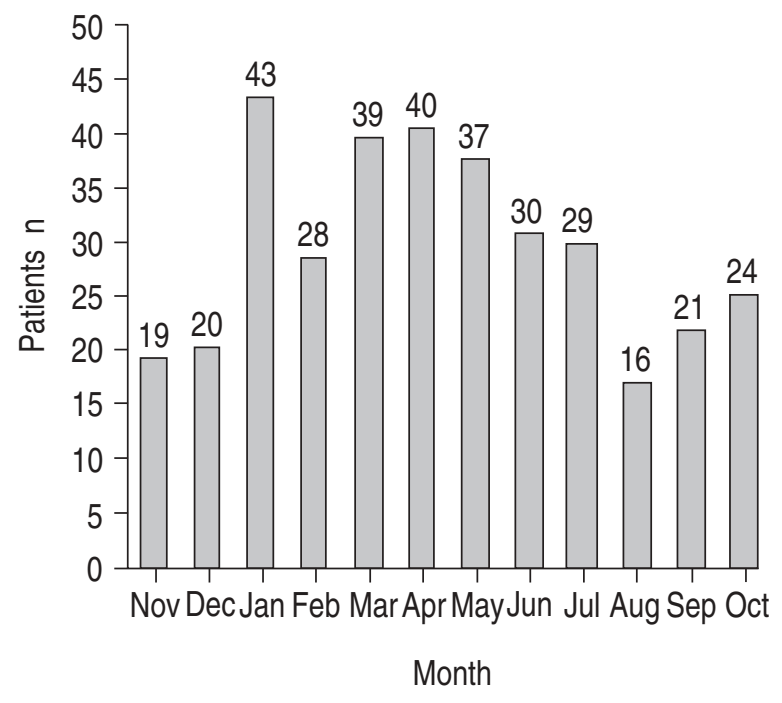

Fig. 1. - The distribution of total community-acquired pnuemonia (CAP) by months of the year.

Figure 1 shows the distribution of all CAP patients by month of the year. The peak month of hospitalization for CAP was January; August had the fewest admissions. The months from March until May had relatively high rates of CAP admissions.

Table 2 shows the frequency distribution of CAP and its primary aetiologies by season. Thirty four percent of the cases occurred in the spring, while only $18 \%$ were seen in the autumn $(\mathrm{p}=0.036)$. Viral aetiology was particularly prevalent in the winter and spring (49 and $37 \%$, respectively), with relatively low incidence in the summer and autumn $(\mathrm{p}=0.009)$. Forty one percent of the cases of M. pneumoniae CAP occurred in the spring compared to $15 \%$ during the winter months. These seasonal differences for $M$. pneumoniae reached borderline statistical significance $(\mathrm{p}=0.054)$. Other aetiological agents such as $S$. pneumoniae, $C$. pneumoniae, Legionella spp., and cases of unknown aetiology did not show significant seasonal variation in their incidence.

Table 3 shows the relative incidence of CAP by aetiology and season. Because of the high rate of multiple

Table 2. - Frequency distribution of total communityacquired pneumonia (CAP) and its aetiologies by seasons

\begin{tabular}{lcccccc}
\hline & & \multicolumn{3}{c}{ Autumn } & Winter & \multicolumn{2}{c}{ Spring } & \multicolumn{2}{c}{ Summer } \\
& $\mathrm{n}$ & $\%$ & $\%$ & $\%$ & p-value* \\
\hline Total CAP & 346 & 18 & 26 & 34 & 22 & 0.036 \\
$\begin{array}{l}\text { Pneumococcus } \\
\text { Mycoplasma }\end{array}$ & 148 & 25 & 20 & 34 & 21 & 0.292 \\
$\begin{array}{l}\text { pneumoniae } \\
\begin{array}{l}\text { Chlamydia } \\
\text { pneumoniae }\end{array}\end{array}$ & 62 & 19 & 15 & 41 & 25 & 0.054 \\
$\begin{array}{l}\text { Legionella } \text { spp. } \\
\text { Respiratory }\end{array}$ & 56 & 27 & 21 & 21 & 30 & 0.888 \\
$\begin{array}{l}\text { viruses } \\
\text { Unknown }\end{array}$ & 35 & 3 & 49 & 37 & 11 & 0.009 \\
aetiology & 67 & 18 & 33 & 33 & 16 & 0.331 \\
\hline
\end{tabular}

*the p-value comparing the observed seasonal distribution of $\mathrm{CAP}$ and each of these aetiologies, with an expected equal seasonal distribution, by Chi-squared test.
Table 3. - Relative incidence of aetiologies of comunity-acquired pneumonia (CAP) as a percentage of total CAP, by seasons*

\begin{tabular}{lcccc}
\hline & $\begin{array}{c}\text { Autumn } \\
(\mathrm{n}=64) \\
\%\end{array}$ & $\begin{array}{c}\text { Winter } \\
(\mathrm{n}=91) \\
\%\end{array}$ & $\begin{array}{c}\text { Spring } \\
(\mathrm{n}=116) \\
\%\end{array}$ & $\begin{array}{c}\text { Summer } \\
(\mathrm{n}=75) \\
\%\end{array}$ \\
\hline Pneumococcus & 58 & 32 & 44 & 41 \\
Mycoplasma pneumoniae & 30 & 16 & 36 & 33 \\
Chlamydia pneumoniae & 17 & 20 & 15 & 21 \\
Legionella spp. & 23 & 13 & 10 & 23 \\
Respiratory viruses & 2 & 19 & 11 & 5 \\
Unknown aetiology & 19 & 24 & 19 & 15 \\
\hline
\end{tabular}

*the sum of percentages for each of the columns is greater than $100 \%$ since some of the patients had multiple aetiologies for CAP.

Table 4. - Mean daily temperatures and relative humidity in Beer-Sheva, Israel during the months of the study

\begin{tabular}{lcc}
\hline Month & $\begin{array}{c}\text { Mean daily } \\
\text { temperature } \\
{ }^{\circ} \mathrm{C}\end{array}$ & $\begin{array}{c}\text { Mean daily } \\
\text { relative humidity } \\
\%\end{array}$ \\
\hline November 1991 & 17.9 & 62.7 \\
December 1991 & 11.1 & 66.5 \\
January 1992 & 9.0 & 68.0 \\
February 1992 & 9.7 & 71.5 \\
March 1992 & 12.8 & 60.6 \\
April 1992 & 17.4 & 56.4 \\
May 1992 & 21.6 & 51.2 \\
June 1992 & 24.9 & 49.2 \\
July 1992 & 25.6 & 55.3 \\
August 1992 & 27.1 & 58.9 \\
September 1992 & 24.5 & 57.9 \\
October 1992 & 23.0 & 59.3 \\
\hline
\end{tabular}

aetiologies, we could not conduct a statistical comparison of seasonal incidence. This also explains why the sum of the percentages is greater than $100 \%$ for each season. It is noteworthy that in the autumn pneumococcal CAP was identified in $58 \%$ of the CAP patients, while this aetiology was found in only $32 \%$ of the patients admitted in the winter. M. pneumoniae was found in only $16 \%$ of the patients diagnosed in the winter. Respiratory viruses were identified in only 2 and $5 \%$ of CAP patients in the autumn and summer, respectively, compared with 19 and $11 \%$ of patients in the winter and spring, respectively.

Table 4 presents data relating to mean daily temperatures and relative humidities in Beer-Sheva during the months the study was conducted.

\section{Discussion}

The seasonal distribution of CAP and the seasonal variation of its aetiological agents, as found in the present study, have three unique features which raise questions as to the general validity of our findings: the CAP patients were all hospitalized; the study was conducted over one year only; and the geographical region is limited. The majority of published studies on CAP, like our own, have reported only on hospitalized patients. Data on CAP patients who were not hospitalized 
is scarce. In retrospect, many of the patients in the present study did not have a clear-cut indication for hospitalization [12]. In this respect, our results also reflect, at least in part, the distribution of nonhospitalized CAP patients.

This study lasted for 1 year, a period of time which enabled us to present the seasonal incidence of CAP and its main aetiologies for that year. An important question is whether the findings of that particular year are representative of other years. Two studies that reported on the incidence of CAP over consecutive years [1, $3]$ showed that peak seasonal incidences are not identical from year to year. These reports oblige us to qualify the generalizability of our data with the statement that the seasonal distribution presented is valid for the study year itself and may not indicate similar incidences for other years.

As the present study was limited to one year, we cannot definitively confirm or negate the possibility that the study period represented an "epidemic period" for at least some of the pathogens. Although analysis of the monthly frequency distribution of each of the aetiological agents negates the possibility that an epidemic lasted for the entire year, it is conceivable that these distributions reflect epidemics lasting for several years, with seasonal outbreaks in certain seasons during each of the epidemic years. This question might have been more effectively addressed, particularly for $M$. pneumoniae, if we had been able to test sera of other populations, that did not have CAP, from this same time period. Unfortunately, such sera was not available to us for testing.

The geographical region in which this study was conducted, i.e. the south of Israel, could also affect the generalizability of our findings, in light of the regional variation which has been reported in the prevalence of various CAP pathogens [11]. The climate in the Negev region of southern Israel is relatively hotter than many other regions of the western world, and could affect the seasonal distribution of CAP as reported here.

Despite the fact that the prevalence of all cases of CAP reached a peak in January, the incidence of CAP in the winter was only $26 \%$ of the rate for the entire year. On the other hand, each of the spring months (March to May) showed relatively high rates of total CAP, so that the incidence of CAP in the spring reached $34 \%$ of the year's incidence. In contrast, each of the monthly incidences in the autumn were relatively low, and the total incidence for this season was only $18 \%$. These seasonal differences were statistically significant. We conclude from these findings and from the meteorological data presented in table 4 that, in contrast with the popularly held view of the general public, the winter with its low temperatures and high humidity is not the main cause of CAP. Similarly, the seasonal incidence found by us does not support the contention that the transition months are "risk periods" for CAP, as they are for allergic disorders, since a particularly low incidence of CAP was found in the autumn. We do not have a convincing explanation for the peak prevalence of CAP in the spring. The meteorological data do not provide an explanation for this peak. The possibility that an epidemic peak of one of the aetiological agents caused the observed peak for all CAP pathogens is not logical, since all the pathogens demonstrated a clear increase in prevalence in the spring months.

MARRIE et al. [2] described 588 CAP patients who were hospitalized over a period of 5 yrs. Analysis of the pooled monthly distribution of cases for the entire study period shows that the seasonal frequencies were almost equal. FRANSEN [3], on the other hand, reports the number of hospitalizations by month for each of the 3 study years. The peak of hospitalizations for CAP was in the spring in 1964, in the late spring and early summer in 1965, and in the winter in 1966. Foy et al. [1] reported the incidence of "total pneumonia" over a consecutive 5 year period. Their report was not restricted to hospitalized patients or to adults. Three of the annual peaks in this report occurred in the spring and two other peaks occurred in the winter. We believe that these series show that there is a seasonal variation in the incidence of total CAP and that in the majority of cases the peak incidence occurs in the spring months, as we also found, although some peaks are also observed in the winter months.

The six respiratory viruses that we investigated as causative agents for CAP had significantly higher incidence in the winter and spring, a low incidence in the summer, and were almost not found in the autumn. This is consistent with data showing that influenza $\mathrm{A}$ and influenza B, which caused most of the respiratory viral infections in the present study, reach peak incidence in the winter and spring [13].

The occurrence of $M$. pneumoniae CAP throughout the year is a well-documented finding in the literature $[1,14-20]$. Seasonal peaks have been reported in only some of these studies. These peaks have been reported in varying periods ranging from the end of summer to winter [14, 16-20]. In the present study, we observed a seasonal peak (with borderline statistical significance) in the spring, with a gradually decreasing incidence as winter approached. The early appearance of a seasonal peak in this study may be due primarily to the relatively warm climate in our region, which is reflected in mean temperatures in the spring which are similar to those measured in the summer months in regions of the world in which the peak occurrence of CAP has been reported to be in the summer.

There was a higher, though not statistically significant, incidence of pneumococcal CAP in the spring compared to the other seasons of the year. This incidence is similar to the higher incidence of pneumococcal infections in the spring observed by FRANSEN [3].

Two important causative agents of CAP, Legionella spp. and $C$. pneumoniae had equal distributions of CAP incidence throughout the year. This is consistent with previous reports concerning CAP caused by $C$. pneumoniae [21, 22] and Legionella spp. [23, 24].

From the point of view of the clinician responsible for the treatment of CAP patients, the seasonal variation of CAP as presented in table 3 (i.e., the relative incidence for each specific aetiology by season) is important. In this method of presentation, one can see that in certain seasons of the year the relative incidence of specific aetiologies is as much as 2 times higher than in other seasons. However, it is difficult to think of practical applications for this method of presentation, 
since the specific frequency for each of the aetiologies is large enough to necessitate appropriate antibiotic therapy at least in the first stage of treatment, considering the large degree of overlap in the clinical manifestations of the various aetiologies of CAP [25].

We conclude that, despite prevailing popular opinion, winter with its low temperatures is not the main explanation for the incidence of community-acquired pneumonia. Specific aetiological causes of communityacquired pneumonia, with the exception of respiratory viruses and Chlamydia pneumoniae, do not show significant seasonal variation.

\section{References}

1. Foy HM, Kenny GE, McMahan R. Mycoplasma pneumoniae pneumonia in an urban area. J Am Med Assoc 1970; 214: 1666-1672.

2. Marrie TJ, Haldane EV, Paulkner RS, et al. Communityacquired pneumonia requiring hospitalization: is it different in the elderly? J Am Geriatr Soc 1985; 33: 671-680.

3 Fransen H. Clinical and laboratory studies on the role of viruses, bacteria, Mycoplasma pneumoniae, and Bedsonia in acute respiratory illness. Scand J Infect Dis 1970; 2 (Suppl 1): 1-38.

4. Jalonen E, Taira S, Paton JC, Kerttula Y, Suomalainen $\mathrm{P}$, Leinonen M. Pneumolysin produced in Bacillus subtilis as antigen for measurement of pneumococcal antibodies by enzyme immunoassay. Serodiagn Immunother Infect Dis 1990; 4: 451-458.

5. Jalonen E, Paton JC, Koskela M, Kerttula Y, Leinonen M. Measurement of antibody responses to pneumolysin: a promising method for the presumptive aetiological diagnosis of pneumococcal pneumonia. J Infect 1989; 19: 127-134.

6. Burman LA, Leinonen M, Trollfors B. Use of serological methods to diagnose pneumonia caused by noncapsulated Haemophilus influenzae and Moraxella catarrhalis. J Infect Dis 1994; 170: 220-222.

7. Leinonen M, Luotonen J, Herva E, Valkonen K, Makela PH. Preliminary serologic evidence for a pathogenic role of Branhamella catarrhalis. J Infect Dis 1981; 144: 570-574.

8. Claesson B, Leinonen M. Moraxella (Branhamella) catarrhalis, an uncommon cause of community-acquired pneumonia in Swedish children. Scand J Infect Dis 1994; 26: 399-402.

9. Leinonen M, Syrjala H, Jalonen E, Kujala P, Herva E. Demonstration of pneumolysin antibodies in circulating immune complexes: a new diagnostic method for pneumococcal pneumonia. Serodiagn Immunother Infect Dis 1990; 4: 451-458.
10. Holloway Y, Snijder JA, Boersma WG. Demonstration of circulating pneumococcal immunoglobulin $\mathrm{G}$ immune complexes in patients with community-acquired pneumonia by means of an enzyme-linked immunosorbent assay. J Clin Microbiol 1993; 31: 3247-3254.

11. Lieberman D, Schlaeffer F, Boldur I, et al. Multiple pathogens in adult patients admitted with communityacquired pneumonia: a one year prospective study of 346 consecutive patients. Thorax 1996; 51: 179-184.

12. Porath A, Schlaeffer F, Lieberman D. Appropriateness of hospitalization of patients with community-acquired pneumonia. Ann Emerg Med 1996; 27: 176-183.

13. Macfarlane J. Community-acquired pneumonia. $\mathrm{Br} \mathrm{J}$ Dis Chest 1987; 81: 116-127.

14. Mansel JK, Rosenow EE, Smith TF, Martin JW. Mycoplasma pneumoniae pneumonia. Chest 1989; 95: 639-646.

15. Atmar RL, Greenberg SB. Pneumonia caused by Mycoplasma pneumoniae and the TWAR agent. Semin Respir Infect 1989; 4: 19-31.

16. Luby JP. Pneumonia caused by Mycoplasma pneumoniae infection. Clin Chest Med 1991; 12: 237-244.

17. Denny FW, Clyde WA, Glezen WP. Mycoplasma pneumoniae disease: clinical spectrum, pathophysiology, epidemiology and control. J Infect Dis 1971; 123: 7492.

18. Levine DP, Lerner AM. The clinical spectrum of Mycoplasma pneumoniae infections. Med Clin North Am 1978; 62: 961-978.

19. Takakura I. Mycoplasmal pneumonia: review of 202 hospitalized cases in Tokai University Hospital from 1975 to 1987. Tokai J Exp Clin Med 1988; 13: 129-135.

20. Johnson DH, Cunha BA. Atypical pneumonias: clinical and extrapulmonary features of Chlamydia, Mycoplasma and Legionella infections. Postgrad Med 1993; 93: 69-82.

21. Kleemola M, Saikku P, Visakorpi R, Wang SP, Grayston JT. Epidemics of pneumonia caused by TWAR, a new Chlamydia organism, in military trainees in Finland. $J$ Infect Dis 1988; 157: 230-236.

22. Saikku P. The epidemiology and significance of Chlamydia pneumoniae. J Infection 1992; 25(Suppl. 1): 27-34.

23. Reboullet V, Gauthier C, Ranfaing J. Comparative epidemiological characteristics of a homogeneous series of acute common pneumopathies seen in a general hospital center Rev Mal Respir 1992; 9: 449-453.

24. Falcó V, Fernandez de Sevilla T, Alegre J, Ferrer A. Martinez-Vazquez JM. Legionella pneumophila, a cause of severe community-acquired pneumonia. Chest 1991; 100: 1007-1111.

25. Fein AM, Niederman MS. Guidelines for initial management of community-acquired pneumonia: savory recipe or cookbook for disaster? Am J Respir Crit Care Med 1995: 152: 1149-1153. 